\title{
USO CORPORATIVO DE MÍDIA SOCIAL E A RESPONSABILIDADE SOCIAL CORPORATIVA
}

\author{
CORPORATE USE OF SOCIAL MEDIA AND CORPORATE SOCIAL RESPONSIBILITY
}

\author{
MIKAÉLI DA SILVA GIORDANI \\ Universidade Regional de Blumenau \\ Titulação: Mestre em Ciências Contábeis \\ Orcid: http://orcid.org/0000-0002-4871-7858 \\ E-mail: mikagiordani@hotmail.com
}

Endereço: Rua Antônio da Veiga, 140 - Itoupava Seca, Blumenau - SC, 89030-903

\author{
CAROLINE KEIDANN SOSCHINSKI \\ Universidade Regional de Blumenau \\ Titulação: Doutoranda em Ciências Contábeis e Administração \\ Orcid: http://orcid.org/0000-0002-0135-0729 \\ E-mail: carolinesoschinski@yahoo.com.br \\ ROBERTO CARLOS KLANN \\ Universidade Regional de Blumenau \\ Titulação: Doutor em Ciências Contábeis e Administração \\ Orcid: http://orcid.org/0000-0002-3498-0938 \\ E-mail: rklann@furb.br
}

\begin{abstract}
RESUMO
Este estudo teve como objetivo investigar a relação entre o uso corporativo de mídias sociais e as práticas de responsabilidade social corporativa (RSC), uma vez que o Facebook e o Twitter se caracterizam como meios menos custosos de divulgação de informações, principalmente aquelas não obrigatórias, como as de RSC. Para isso, realizou-se uma pesquisa descritiva, documental e de abordagem quantitativa, por meio da operacionalização de regressão linear múltipla, modelo Tobit, por erros-padrão robustos. A análise consistiu em 360 observações durante os anos de 2013 a 2017. Os principais achados evidenciaram uma relação positiva entre o uso corporativo de mídias sociais e as práticas de RSC, o que foi confirmado pelo teste de sensibilidade. Além disso, as variáveis de controle tamanho, crescimento das vendas, retorno sobre os ativos e alavancagem também se apresentaram significantes, sendo úteis para explicar a RSC. Os resultados deste estudo sugerem que a mídia social e, mais especificamente o Facebook e o Twitter, podem ser considerados meios facilitadores para as empresas divulgarem informações voluntárias, visto que esses canais possibilitam divulgação rápida, abrangente e de menor custo. Portanto, a pesquisa contribui ao expandir a literatura sobre os temas, por meio de testes empíricos, que consideraram medidas agregadas tanto do uso corporativo de mídia social como de RSC.
\end{abstract}

Palavras-chave: Mídia social. Disclosure voluntário. Responsabilidade Social Corporativa. Facebook. Twitter.

Data de submissão: 03/10/2019. Data de aceite: 30/11/2019. Data de publicação: 17/12/2019.

Artigo selecionado no fast track do COGECONT - International Conference in Management and Accounting, realizado na Universidade Comunitária da Região de Chapecó - Unochapecó, Chapecó - SC, de 17 a 19/10/2019. 


\begin{abstract}
This study aimed to investigate the relationship between corporate use of social media and corporate social responsibility (CSR) practices, once Facebook and Twitter are characterized as less costly means of disclosing information, especially non-mandatory ones, like CSR. For this, a descriptive, documentary and quantitative research were carried out through the operation of multiple linear regression, Tobit model, for robust standard errors. The analysis consisted of 360 observations from 2013 to 2017. The main findings showed a positive relationship between corporate use of social media and CSR practices, which was confirmed by the sensitivity test. In addition, the variables of size control, sales growth, return on assets and leverage were also significant, being useful to explain CSR. The results of this study suggest that social media, and more specifically Facebook and Twitter, may be considered as facilitating means for companies to disclose voluntary information, as these channels enable rapid, comprehensive and cost-effective disclosure. Therefore, the research contributes by expanding the literature on the themes through empirical tests that considered aggregate measures of both corporate social media use and CSR.
\end{abstract}

Keywords: Social Media. Voluntary Disclosure. Corporate Social Responsibility. Facebook. Twitter.

\title{
1 INTRODUÇÃO
}

A globalização e o desenvolvimento tecnológico provocaram mudanças no nível social, político e econômico, de forma a contribuir para o surgimento de um novo modelo de sociedade, com novos atores e modelos comportamentais. Diante disso, emergiram expectativas que envolvem a demanda por práticas de responsabilidade social e de um relacionamento cada vez mais transparente entre organizações e sociedade. Assim, destacase o papel das empresas em adotar uma abordagem proativa de responsabilidade social corporativa (RSC) (ALI; JIMENEZ-ZARCO; BICHO, 2015), denominada nesta pesquisa também como disclosure voluntário.

A demanda por informações sobre o comportamento socialmente responsável das organizações, de acordo com a revisão da literatura de Ali, Frynas e Mahmood (2017), reflete as preocupações das partes interessadas no que concerne as responsabilidades das organizações em zelar por um bom relacionamento e pela transparência de informações. Para os autores, órgãos reguladores, ambientalistas, credores e meios de comunicação são os principais influenciadores para o disclosure voluntário de práticas sociais e ambientais.

Por mais que a literatura tenha identificado uma maior atenção às práticas de RSC (ZHAO; ZHANG; KWON, 2018), ainda assim, as empresas têm resistido a adotar políticas de disclosure voluntário. De acordo com Paulino, Silva e Girão (2018), isso se deve à questão dos custos envolvidos nesse processo. De acordo com Dye (2001), há uma relação negativa entre os custos de divulgação e o disclosure voluntário, de forma que os excessivos custos em divulgar tais informações acabam fazendo com que as organizações decidam por não as divulgar ou divulgar com menor intensidade.

Contudo, decorrente dos avanços tecnológicos, as mídias sociais têm se mostrado um meio facilitador para as organizações divulgarem informações voluntárias, pois possibilitam uma comunicação de forma rápida, abrangente e com menor custo de divulgação (BLANKESPOOR; MILLER; WHITE, 2013). Seu uso tem feito com que consumidores e demais usuários das informações não sejam mais indivíduos isolados, ao contrário, estejam conectados uns com os outros e ativos em fornecer feedback útil às empresas (PRAHALAD; 
RAMASWAMY, 2004). Conforme Ghoul et al. (2019), a mídia social pode desempenhar um papel fundamental para estimular o comportamento corporativo responsável.

Quanto às redes de comunicação Facebook e Twitter, podem ser consideradas plataformas eficazes para disseminar informações e proporcionar o engajamento de partes interessadas (CRISAN; ZBUCHEA, 2015), além de influenciar ativamente o comportamento das empresas (GHOUL et al., 2019). Outra vantagem dessas plataformas é a de proporcionar uma comunicação entre os usuários, a qual permite que empresas informem seus stakeholders e que stakeholders, por outro lado, possam responder às organizações, por meio de comentários, curtidas ou tweets (ALI; JIMENEZ-ZARCO; BICHO, 2015).

Por mais que o campo de estudos que tem analisado o uso corporativo das mídias sociais ainda se mostre incipiente - visto que o uso desta ferramenta pelas organizações é uma prática recente (CAO et al., 2018) - algumas pesquisas já investigaram a associação entre o uso corporativo de mídias sociais e o mercado de capitais, sugerindo que o uso dessas plataformas pode impactar no preço dos ativos, na liquidez e na assimetria de informações (BLANKESPOOR; MILLER; WHITE, 2013; CADE, 2018).

Em relação ao uso corporativo de mídia social como meio de divulgação de informações voluntárias, como de RSC, foram identificados estudos como o de Manetti e Bellucci (2016), que avaliou a interação das partes interessadas na organização para definir os conteúdos de relatórios sociais, ambientais ou de sustentabilidade; e de Lodhia e Stone (2017), que investigou o uso de plataformas de mídia social como forma de comunicar às partes externas sobre os relatórios integrados, sendo ambos qualitativos. No âmbito nacional, destaca-se o estudo de Paulino, Silva e Girão (2018), os quais analisaram a relação entre a divulgação voluntária, através das mídias sociais, e a demanda dos investidores por informações de RSC nas empresas pertencentes ao Índice Brasil IBrX 100. Contudo, os autores evidenciaram resultados contrários ao esperado, ao abordar a relação entre o número de seguidores e as informações voluntárias de RSC.

Ao considerar a existência de poucos estudos que buscaram identificar como as empresas utilizam as mídias sociais (MILLER; SKINNER, 2015) e, mais especificamente, os estudos que analisaram as mídias sociais como forma de divulgação de informações voluntárias de RSC (MANETTI; BELLUCI, 2016; LODHIA; STONE, 2017), além de considerar as contradições entre resultados empíricos e preceitos teóricos (PAULINO; SILVA; GIRÃO, 2018), evidenciou-se uma lacuna de pesquisa sobre a relação entre o engajamento corporativo em mídias sociais e as práticas de RSC. Assim, formulou-se a seguinte questão norteadora desta pesquisa: Qual a relação entre o uso corporativo das mídias sociais e as práticas de RSC? Para tanto, este estudo objetiva investigar se empresas mais engajadas no uso corporativo do Facebook e do Twitter apresentam maiores práticas de RSC.

De acordo com Lodhia e Stone (2017), é necessário novas pesquisas para avaliar até que ponto o potencial das mídias sociais é realmente utilizado pelas organizações e qual a relação com práticas voluntárias, como as de RSC. Adicionalmente, Manetti e Bellucci (2016) relatam que pesquisas adicionais podem complementar os estudos sobre os temas, por meio de pesquisas exploratórias com análises estatísticas. Dessa forma, o presente estudo se justifica por buscar evidências quantitativas adicionais a estas temáticas.

Esta pesquisa se diferencia dos estudos de Manetti e Bellucci (2016) e Lodhia e Stone (2017) por abordar uma investigação longitudinal, e ao contrário de Paulino, Silva e Girão (2018), que analisaram apenas o ano de 2016. Além disso, este estudo demonstra resultados que expandem os de Paulino, Silva e Girão (2018), visto que os autores evidenciaram resultados contrários aos preceitos teóricos estabelecidos pela literatura, enquanto este 
estudo, por outro lado, encontrou resultados condizentes com a predição teórica, o que revela que empresas brasileiras com maior engajamento em mídias sociais também possuem maiores índices de disclosure voluntário.

Esta pesquisa contribui para a literatura sobre disclosure voluntário, especialmente sobre RSC, ao investigar a relação entre o engajamento das partes interessadas na organização por meio de mídias sociais e as práticas de RSC, o que complementa pesquisas anteriores que já analisaram esta relação por meio de investigações transversais e utilizando outras variáveis de RSC. A contribuição também está voltada à literatura de contabilidade social e ambiental, ao determinar que as mídias sociais podem atuar como instrumentos confiáveis de engajamento efetivo das partes interessadas para promoção da RSC.

\section{ANTECEDENTES E HIPÓTESE DE PESQUISA}

As plataformas de redes sociais são particularmente adequadas para o engajamento das partes interessadas, pois, o elemento comunitário incorporado dentro delas permite interagir com um grande grupo de pessoas (MANETTI; BELLUCCI, 2016). Além disso, as mídias sociais permitem que os usuários troquem informações em tempo hábil e busquem e forneçam feedback rápido através de posts e diálogo em tempo real (LODHIA; STONE, 2017).

As mídias sociais podem ser usadas para gerar informações e para interação social. Dessa forma, a capacidade das partes interessadas de responder e comentar as postagens das mídias sociais e dialogar com outros usuários online eleva ainda mais o processo de comunicação entre organização e stakeholders. No entanto, o engajamento e a reação do mercado à plataforma de mídia social dependem do nível de interação e das características da empresa (CHAHINE; MALHOTRA, 2018).

As mídias sociais têm amplo impacto em todas as esferas do desempenho dos negócios, como finanças, operações e desempenho social corporativo. Especificamente, Paniagua e Sapena (2014) identificaram quatro canais distintos pelos quais as mídias sociais afetam o desempenho dos negócios, os quais correspondem ao capital social, marketing, rede corporativa e preferências reveladas pelos clientes. Cada esfera compreende um conjunto de recursos de mídias sociais que causa implicações para as empresas.

No contexto da presente pesquisa, destaca-se dois canais abordados por Paniagua e Sapena (2014). Primeiro: o canal do capital social, que se refere ao desempenho social corporativo, representa a medida em que as mídias sociais afetam as relações das empresas com a sociedade. Segundo: o canal de preferências reveladas, que representa a extensão em que as mídias sociais expõem os gostos dos clientes, por meio de conversas e compartilhamentos, o que, consequentemente, impacta nas ações organizacionais.

A utilização de mídias sociais contribui para as empresas ao apoiar a criação de relacionamento com os clientes (AININ et al., 2015; HE; WANG; AKULA, 2017; AGOSTINO; SIDOROVA, 2017) e demais interessados na organização. Dessa forma, as informações fornecidas nessas plataformas possibilitam que as empresas se concentrem nas necessidades e preocupações dos clientes, tornando-se mais competitivas, o que também resulta em um melhor desempenho organizacional (RODRIGUEZ; PETERSON; AJJAN, 2015).

Outro ponto positivo, constatado na literatura sobre mídias sociais, corresponde à redução da distância organizacional entre gerentes e funcionários. A inclusão de plataformas de mídia social na organização faz com que os funcionários se sintam parte do processo de tomada de decisão da empresa, o que aumenta sua motivação e influência no desempenho organizacional (FERRER et al., 2013). 
Em relação às partes externas interessadas na organização, estas verificam a presença da empresa nas plataformas de mídia social e o que os clientes estão dizendo sobre ela e, assim, usam esse feedback para obter informações sobre onde e o que investir (CHANDA; ZAORSKI, 2013). Portanto, infere-se que as organizações são incentivadas a usar as mídias sociais também como uma forma de se manterem competitivas no mercado de capitais.

Além disso, há evidências de que organizações utilizam as mídias sociais como forma de divulgar informações voluntárias, como as de RSC, com o intuito de interagir e informar suas partes interessadas, visto que a divulgação mediante mídias sociais possui um baixo custo e permite a comunicação entre empresas e stakeholders (BLANKESPOOR; MILLER; WHITE, 2013; ALI; JIMENEZ-ZARCO; BICHO, 2015). Contudo, de acordo com Manetti e Bellucci (2016), a utilização da mídia social para o disclosure voluntário ainda não é uma prática comum entre as organizações que publicam informações sobre RSC.

Tratando-se do disclosure voluntário de informações em mídias sociais, destaca-se a pesquisa nacional de Paulino, Silva e Girão (2018), que verificou a influência do número de seguidores e o número de curtidas do Facebook e do Twitter na RSC, em empresas pertencentes ao $\mathrm{IBrX} 100$. Os autores sustentaram teoricamente uma influência positiva nas mídias sociais, tanto do número de seguidores como do número de curtidas. Essa expectativa foi confirmada ao tratar sobre a relação entre o número de curtidas e o disclosure voluntário, contudo, a demanda dos usuários por informações corporativas via mídias sociais (número de seguidores) apresentou uma relação negativa com o disclosure voluntário de informações ambientais e sociais, ao contrário do sustentado pela literatura.

De acordo com Drake, Roulstone e Thornock (2012) e Paulino, Silva e Girão (2018), o uso corporativo de mídia social possibilita o engajamento das partes interessadas e, portanto, entende-se que a demanda de informações, medidas pelo número de seguidores, impulsione as organizações a divulgarem mais informações de RSC, visto que o Facebook e o Twitter são considerados canais de comunicação de baixo custo, direto e tempestivo. Baseando-se nesses preceitos teóricos, elaborou-se a seguinte hipótese de pesquisa:

$\mathrm{H}_{1}$ : O uso corporativo de mídia social está positivamente relacionado com a divulgação de práticas de responsabilidade social corporativa em empresas brasileiras.

\section{PROCEDIMENTOS METODOLÓGICOS}

Esta pesquisa se caracteriza como documental, descritiva e com abordagem quantitativa dos dados. A população foi identificada pelas empresas listadas na Brasil, Bolsa e Balcão $[B]^{3}$ no período de 2018. A amostra, por sua vez, foi delineada com base nas empresas que possuíam informações para o cálculo das variáveis. Ressalta-se que o fato de as informações sobre RSC serem informações não obrigatórias, apenas um número limitado de empresas as divulga, o que, consequentemente, torna a amostra desta pesquisa limitada e desbalanceada, visto que as empresas que divulgam RSC em um ano não necessariamente divulgam no ano seguinte. Desta forma, esta pesquisa conta com 360 observações, durante os anos de 2013 a 2017.

Inicialmente, foram identificadas as empresas que possuíam score de RSC, as quais, conforme a Tabela 1, representam, em média, 72 empresas por ano. Posteriormente, foram identificadas se estas possuíam mídia social (Facebook e Twitter). A escolha destas mídias sociais se justifica pela possibilidade de diferentes formas de publicação, como imagens, textos e vídeos; e interação, por meio de comentários, curtidas, compartilhamentos e retweets (Jung et al., 2018), o que consequentemente, facilita o engajamento, tornando-se, assim, canais de menor custo e adequados para o uso corporativo. Ainda segundo Manetti, 
Belluci e Bagnoli (2017), Jung et al. (2018) e Dlamini e Johnston (2018), o Facebook e o Twitter são as plataformas de mídia social mais adotadas pelas empresas.

O procedimento de verificação da mídia social seguiu a metodologia abordada por Jung et al. (2018). Primeiramente, foi realizado o acesso ao website corporativo de cada empresa para evidenciar se este possuía link que direcionasse à mídia social. Esse procedimento é fundamental, pois, garante que a mídia social verificada seja a oficial da empresa. Após essa constatação, foi realizado o acesso ao Facebook e Twitter corporativo das empresas que possuem a mídia e coletado os dados. No Twitter, os dados correspondem ao número total de tweets, número de usuários que seguem a empresa, número de usuários que a empresa segue, número de curtidas na mídia e ano em que a empresa aderiu à mídia social. No Facebook, referem-se ao número de seguidores e curtidas na página. Nesta pesquisa, o uso corporativo das mídias sociais é indicado por meio do engajamento corporativo na média dos indicadores supracitados, sendo que maiores números indicam um maior engajamento.

A Tabela 1 apresenta a amostra de pesquisa dividida entre empresas com e sem informações referentes às mídias sociais, durante os anos de análise.

Tabela 1 - Empresas com e sem mídia social por período

\begin{tabular}{c|c|c|c|c|c}
\hline Ano & $\begin{array}{c}\text { Empresas com } \\
\text { Facebook }\end{array}$ & $\begin{array}{c}\text { Empresas sem } \\
\text { Facebook }\end{array}$ & $\begin{array}{c}\text { Empresas com } \\
\text { Twitter }\end{array}$ & $\begin{array}{c}\text { Empresas sem } \\
\text { Twitter }\end{array}$ & Amostra \\
\hline 2013 & 50 & 23 & 40 & 33 & 73 \\
2014 & 50 & 24 & 40 & 34 & 74 \\
2015 & 50 & 25 & 40 & 35 & 75 \\
2016 & 49 & 26 & 40 & 35 & 75 \\
2017 & 41 & 22 & 35 & 28 & 63 \\
\hline Observações & 240 & 120 & 195 & 165 & 360 \\
\hline
\end{tabular}

Fonte: Dados da pesquisa.

Dentre as empresas que possuem disclosure ambiental, em relação ao Facebook, observa-se que, em média, cerca de 48 (24) possuem (não possuem) esta mídia social, enquanto 39 empresas, em média, adotam o Twitter. Percebe-se que o número de empresas que possuem a mídia social Facebook é superior àquelas que adotam o Twitter. Ressalta-se que as empresas que não possuíam Facebook ou Twitter durante o período de coleta foram mantidas na pesquisa, como forma de representar o grupo de controle.

As variáveis de RSC e as informações financeiras para cálculo das variáveis de controle foram coletadas na base de dados da Thomson Reuters Eikon. O Quadro 1 demonstra as variáveis utilizadas neste estudo.

Ressaltam-se alguns detalhes pertinentes sobre as variáveis apresentadas no Quadro 1. As variáveis que compõem a RSC ( RSC $_{i t}$ ) são dados secundários, visto que foram coletadas no relatório ESG (Environmental, Social and Governance), disponível na base de dados da Thomson Reuters Eikon. Neste relatório estão disponíveis duas medidas, denominadas como o pilar ambiental e social, os quais são compostos por 3 e 4 dimensões, respectivamente. 0 pilar ambiental da RSC é composto pelas dimensões de uso de recursos, emissão de poluentes e poluição, enquanto o pilar social da RSC é composto pelas dimensões de força de trabalho, direitos humanos, comunidade e responsabilidade com os produtos.

O pilar social mede a capacidade das empresas em gerar confiança e fidelidade com sua força de trabalho, clientes e sociedade, através do uso de melhores práticas de gestão. 0 pilar ambiental, por sua vez, mede o impacto das empresas em sistemas naturais vivos e não vivos, incluindo o ar, a terra e a água, bem como ecossistemas completos. Esse pilar reflete o quão bem uma empresa usa as práticas de gestão para evitar riscos ambientais e capitalizar as oportunidades ambientais, a fim de gerar valor para acionistas no longo prazo. 
EDIÇÃO ESPECIAL: COMEMORATIVA AOS 45 ANOS DOS CURSOS DE GRADUAÇÃO EM CIÊNCIAS CONTÁBEIS E ADMINISTRAÇÃO DA UNOCHAPECÓ

Quadro 1 - Variáveis da pesquisa

\begin{tabular}{|c|c|c|c|c|}
\hline \multicolumn{2}{|c|}{ Variáveis/ Definição } & Fórmula & Coleta & Autores \\
\hline \multicolumn{5}{|c|}{ Variáveis dependentes } \\
\hline \multirow{2}{*}{$\mathrm{RSC}_{\mathrm{it}}$} & Pilar Social & \multirow{2}{*}{$\begin{array}{l}\text { Score de } 0 \text { a } 100 \text { : Quanto mais } \\
\text { itens as empresas divulgam, } \\
\text { mais próximo a } 100 \text { será sua } \\
\text { pontuação. }\end{array}$} & \multirow{2}{*}{$\begin{array}{l}\text { Thomson } \\
\text { Reuters } \\
\text { Eikon }\end{array}$} & Ghoul et al. (2019) \\
\hline & Pilar Ambiental & & & Ghoul et al. (2019) \\
\hline \multicolumn{5}{|c|}{ Variáveis independentes } \\
\hline \multirow{7}{*}{$\mathrm{MS}_{\text {it }}$} & $\begin{array}{l}\text { Usuários que } \\
\text { seguem a empresa }\end{array}$ & $\begin{array}{c}\text { Log natural do número de } \\
\text { usuários que seguem a } \\
\text { empresa }\end{array}$ & \multirow{2}{*}{ Facebook } & $\begin{array}{l}\text { Zhang (2015); Manetti e } \\
\text { Belluci (2016); Manetti, } \\
\text { Belluci e Bagnoli (2017). }\end{array}$ \\
\hline & $\begin{array}{c}\text { Usuários que curtem } \\
\text { a empresa }\end{array}$ & $\begin{array}{l}\text { Log natural do número de } \\
\text { usuários que curtem a página } \\
\text { da empresa }\end{array}$ & & $\begin{array}{l}\text { Zhang (2015); Manetti e } \\
\text { Belluci (2016); Manetti, } \\
\text { Belluci e Bagnoli (2017). }\end{array}$ \\
\hline & Tweets & $\begin{array}{l}\text { Log natural do número total de } \\
\text { tweets }\end{array}$ & \multirow{5}{*}{ Twitter } & $\begin{array}{l}\text { Lee, Hutton e Shu (2015); } \\
\text { Manetti e Belluci (2016); } \\
\text { Manetti, Belluci e Bagnoli } \\
\text { (2017); Jung et al. (2018) }\end{array}$ \\
\hline & $\begin{array}{l}\text { Usuários que } \\
\text { seguem a empresa }\end{array}$ & $\begin{array}{l}\text { Log natural do número de } \\
\text { usuários que seguem a } \\
\text { empresa }\end{array}$ & & $\begin{array}{c}\text { Zhang (2015); Manetti e } \\
\text { Belluci (2016); Manetti, } \\
\text { Belluci e Bagnoli (2017); } \\
\text { Jung et al. (2018) }\end{array}$ \\
\hline & $\begin{array}{l}\text { Usuários que a } \\
\text { empresa segue }\end{array}$ & $\begin{array}{l}\text { Log natural do número de } \\
\text { usuários que a empresa segue }\end{array}$ & & $\begin{array}{l}\text { Manetti e Belluci (2016); } \\
\text { Manetti, Belluci e Bagnoli } \\
\text { (2017); Jung et al. (2018) }\end{array}$ \\
\hline & $\begin{array}{l}\text { Usuários que curtem } \\
\text { a empresa }\end{array}$ & $\begin{array}{l}\text { Log natural do número de } \\
\text { usuários que curtem a } \\
\text { empresa }\end{array}$ & & $\begin{array}{l}\text { Zhang (2015); Manetti e } \\
\text { Belluci (2016); Manetti, } \\
\text { Belluci e Bagnoli (2017); } \\
\text { Jung et al. (2018) }\end{array}$ \\
\hline & $\begin{array}{c}\text { Quantidade de anos } \\
\text { que a empresa } \\
\text { utiliza Twitter }\end{array}$ & $\begin{array}{l}\text { Número de anos que a } \\
\text { empresa usa a mídia social }\end{array}$ & & $\begin{array}{l}\text { Zhang (2015); Jung et al. } \\
\text { (2018) }\end{array}$ \\
\hline \multicolumn{5}{|c|}{ Variáveis Independentes de Controle } \\
\hline TAM $_{\text {it }}$ & Tamanho & Log do ativo total & \multirow{5}{*}{$\begin{array}{l}\text { Thomson } \\
\text { Reuters } \\
\text { Eikon }\end{array}$} & $\begin{array}{c}\text { Paulino, Silva e Girão } \\
\text { (2018); Ghoul et al. (2019) }\end{array}$ \\
\hline $\mathrm{CV}_{\text {it }}$ & $\begin{array}{l}\text { Crescimento das } \\
\text { vendas }\end{array}$ & $\frac{\text { Receita }_{i t}-\text { Receita }_{i t-1}}{\text { Receita }_{i t-1}}$ & & Ghoul et al. (2019) \\
\hline $\mathrm{ROA}_{\mathrm{it}}$ & $\begin{array}{l}\text { Retorno sobre os } \\
\text { ativos }\end{array}$ & $\frac{E_{\text {BIT }}}{\text { AtivoTotal }_{i t}}$ & & Ghoul et al. (2019) \\
\hline $\mathrm{ALA}_{\mathrm{it}}$ & Alavancagem & $\frac{\text { PassivoTotal }_{i t}}{\text { AtivoTotal }_{i t}}$ & & $\begin{array}{c}\text { Chakroun, Matoussi e } \\
\text { Mbirki (2017); Paulino, Silva } \\
\text { e Girão (2018) }\end{array}$ \\
\hline $\mathrm{GC}_{\text {it }}$ & $\begin{array}{l}\text { Governança } \\
\text { Corporativa }\end{array}$ & $\begin{array}{l}\text { Score de } 0 \text { a 100: quanto mais } \\
\text { itens as empresas divulgam, } \\
\text { mais próximo a } 100 \text { será sua } \\
\text { pontuação. }\end{array}$ & & Ghoul et al. (2019); \\
\hline
\end{tabular}

Legenda: RSC it: Responsabilidade Social Corporativa; MS it: Mídia Social; TAMit: Tamanho; $\mathrm{CV}_{\mathrm{it}}$ : Crescimento das Vendas; ROA it: Rentabilidade sobre o Ativo; ALA it: Alavancagem; $\mathrm{GC}_{\text {it: }}$ Governança Corporativa.

Fonte: Dados da pesquisa.

Cada uma das dimensões contempla inúmeros itens, os quais a base de dados analisa se a empresa possui ou não determinada prática. Por exemplo, a dimensão de comunidade é composta por 28 itens que tratam sobre se a empresa tem ou não: políticas contra suborno e corrupção, de justa concorrência, de ética empresarial, de envolvimento comunitário, entre 
outros. Além destes, a base de dados analisa itens monetários, como por exemplo, o valor anual que a empresa destina a doações.

Tanto o pilar social como o ambiental possuem pontuações que variam de 0 a 100 , sendo que quanto mais próximo a 100 , significa que a empresa se engaja em mais práticas socialmente responsáveis. No relatório ESG, foram coletadas as pontuações social e ambiental e, para a operacionalização desta pesquisa, calculou-se a média destas duas, como forma de gerar um score geral de RSC.

Referente às informações do Facebook (usuários que seguem a empresa e usuários que curtem a empresa) e Twitter (tweets, usuários que seguem a empresa, usuários que a empresa segue, usuários que curtem a empresa e a quantidade de anos que a empresa utiliza o Twitter), ressalta-se que estas são medidas (exceto o tempo em que a empresa utiliza a mídia) estáticas (invariantes no tempo), porque os dados da série temporal sobre estes números para cada empresa não estão disponíveis. Entretanto, o estudo realizado por Jung et al. (2018) constatou que as empresas apresentam aumentos consistentes em relação a essas informações, sendo que, dessa forma, o uso de uma contagem estática não impulsiona os resultados.

Para o desenvolvimento da variável mídia social, considerou-se as variáveis acima citadas do Facebook e do Twitter para construir um score de uso corporativo de cada uma dessas mídias ( $\mathrm{MS}_{\mathrm{it}}$ ). Para isso, o método Multi-Objective Optimization on the basis of Ratio Analisys (MOORA) foi operacionalizado com os dados das empresas que possuem Facebook e Twitter. O método MOORA é uma ferramenta de análise decisória multicritério, cujo cálculo foi realizado da seguinte forma: o conjunto de dados foi elevado ao quadrado e dividido pela soma dos dados ao quadrado como denominadores, os índices utilizados foram situados entre zero e um e, por fim, foram adicionados (BRAUNERS; ZAVADSKAS, 2006), sendo que, quanto maior o índice, maior o uso corporativo da mídia social.

Baseando-se na pesquisa de Ghoul et al. (2019) para a escolha das variáveis de controle, utilizou-se o tamanho medido pelo ativo total (TAM ${ }_{i t}$ ), o crescimento das vendas $\left(\mathrm{CV}_{i t}\right)$, a rentabilidade sobre $\mathrm{o}$ ativo $\left(R O A_{i t}\right)$, a alavancagem $\left(A L A_{i t}\right)$ e a governança corporativa $\left(G C_{i t}\right)$. Ressalta-se que a variável de $G C$ mede os sistemas e processos das empresas, assegurando que membros e executivos atuem no melhor interesse de seus acionistas de longo prazo. Essa medida reflete a capacidade das organizações de direcionar e controlar os direitos e responsabilidades, por meio das melhores práticas de gestão (incentivos). Essa medida varia de 0 a 100, sendo que quanto mais próximo a 100 mais a empresa atende aos itens de GC analisados pela base de dados da Thomson Reuters Eikon.

Os dados foram operacionalizados por meio de regressão linear múltipla, modelo Tobit, devido a variável dependente ser censurada (de 0 a 100), com efeito fixo de setor e ano. Os setores de atuação das empresas foram classificados de acordo com o North American Industry Classification System (NAICS). O modelo econométrico testado é baseado no engajamento dos stakeholders para evidenciar a relação entre as mídias sociais e a RSC, conforme apresentado na Equação 1:

$$
\begin{gathered}
R S C_{i t}=\beta_{0}+\beta_{1} M S_{i t}+\beta_{2} T_{A M}+\beta_{3} C V_{i t}+\beta_{4} R O A_{i t}+\beta_{5} A L A_{i t}+\beta_{6} G C_{i t}+ \\
\text { EfeitosFixosSetor }+ \text { EfeitosFixosAno }+\varepsilon
\end{gathered}
$$

Equação 1

Destaca-se que a Equação 1 foi operacionalizada separadamente para cada mídia social, bem como, com e sem variáveis de controle. Os pressupostos dos modelos de regressão linear múltipla foram testados e estão apresentados na análise dos resultados. Salienta-se, no 
entanto, que possíveis problemas de heterocedasticidade foram solucionados mediante a utilização de erros-padrão robustos.

\section{APRESENTAÇÃO E ANÁLISE DOS RESULTADOS}

Essa seção é destinada a apresentação e análise dos resultados. Inicialmente, apresenta-se a estatística descritiva das variáveis de pesquisa, bem como o teste $t$ de médias para verificar diferenças entre o grupo de empresas que possui mídia social ou não. Posteriormente, apresenta-se a matriz de correlação e, por fim, os resultados das regressões que visam atender ao objetivo proposto nesta pesquisa, ou seja, o de investigar a relação entre mídia social e RSC.

As variáveis de rentabilidade sobre o ativo $\left(R O A_{i t}\right)$, alavancagem ( $\left(A L A_{i t}\right)$ e crescimento das vendas $\left(\mathrm{CV}_{\text {it }}\right)$ foram operacionalizadas em seus valores winsorizados a $1 \%$. As demais variáveis não foram winsorizadas, devido ao fato de que a RSC e GC são expressas em medidas que variam de 0 a 100, o tamanho foi logaritmizado e a mídia social foi construída com base no método MOORA. Portando, ROA, ALA e CV são as únicas que não passaram por um processo de padronização anterior.

Na Tabela 2, Painel A e B, apresenta-se o teste t para comparar o grupo de empresas com e sem mídia social. No Painel C, apresenta-se a estatística descritiva das variáveis.

Tabela 2 - Estatística descritiva das variáveis

\begin{tabular}{l|c|c|c|c|c|c}
\hline \multicolumn{2}{l}{ Painel A - Teste t de médias entre grupo de empresas com e sem Facebook } \\
\hline & \multicolumn{2}{|c|}{ Com Mídia Social } & \multicolumn{2}{c|}{ Sem Mídia Social } & \multicolumn{2}{c}{ Teste t de Student } \\
\hline & Média & Desvio Padrão & Média & Desvio Padrão & t & Sig. \\
\hline RSC $_{i t}$ & 61,3676 & 15,7034 & 44,4411 & 18,7675 & $-9,020$ & $0,001^{* * *}$ \\
TAM $_{i t}$ & 22,4786 & 1,2919 & 21,9539 & 1,1658 & $-3,750$ & 0,157 \\
CV $_{i t}$ & 0,0837 & 0,3978 & 0,2721 & 1,1807 & 2,234 & $0,000^{* * *}$ \\
ROA $_{i t}$ & 0,0781 & 0,0731 & 0,0445 & 0,1317 & $-3,112$ & $0,004^{* * *}$ \\
ALA $_{i t}$ & 0,6243 & 0,1985 & 0,5853 & 0,3173 & $-1,425$ & $0,000^{* * *}$ \\
GC $_{i t}$ & 53,3481 & 19,1316 & 47,883 & 18,7193 & $-2,573$ & 0,960 \\
\hline
\end{tabular}

Painel B - Teste t de médias entre grupo de empresas com e sem Twitter

\begin{tabular}{|c|c|c|c|c|c|c|}
\hline & \multicolumn{2}{|c|}{ Com Mídia Social } & \multicolumn{2}{|c|}{ Sem Mídia Social } & \multicolumn{2}{|c|}{ Teste t de Student } \\
\hline & Média & Desvio Padrão & Média & Desvio Padrão & $\mathrm{t}$ & Sig. \\
\hline $\mathrm{RSC}_{\text {it }}$ & 62,7247 & 15,1430 & 47,4536 & 18,8774 & $-8,514$ & $0,000 * * *$ \\
\hline TAM $_{\text {it }}$ & 22,6927 & 1,2886 & 21,8440 & 1,0936 & $-6,668$ & $0,000 * * *$ \\
\hline $\mathrm{CV}_{\text {it }}$ & 0,0803 & 0,2669 & 0,2247 & 1,0789 & 1,806 & $0,072^{*}$ \\
\hline $\mathrm{ROA}_{\text {it }}$ & 0,0805 & 0,0691 & 0,0508 & 0,1216 & $-2,195$ & $0,029 * *$ \\
\hline $\mathrm{ALA}_{\mathrm{it}}$ & 0,6309 & 0,2055 & 0,5881 & 0,2833 & $-1,654$ & 0,099* \\
\hline$G C_{i t}$ & 55,9392 & 18,3198 & 46,3117 & 18,8338 & $-4,893$ & $0,000 * * *$ \\
\hline
\end{tabular}

Painel C - Estatística descritiva

\begin{tabular}{|c|c|c|c|c|c|}
\hline & Média & Desvio Padrão & Percentil 25 & Mediana & Percentil 75 \\
\hline$M S \_F B_{i t}$ & 0,0841 & 0,0628 & 0 & 0,1139 & 0,1304 \\
\hline MS_TW & 0,3198 & 0,3434 & 0 & 0,3628 & 0,5738 \\
\hline $\mathrm{RSC}_{\text {it }}$ & 55,7254 & 18,5679 & 41,5883 & 59,2515 & 71,9338 \\
\hline TAM $_{i t}$ & 22,3037 & 1,2739 & 21,5375 & 22,2524 & 23,0995 \\
\hline $\mathrm{CV}_{\text {it }}$ & 0,1465 & 0,7586 & $-0,0443$ & 0,0671 & 0,1496 \\
\hline ROA $_{i t}$ & 0,0669 & 0,0977 & 0,0350 & 0,0630 & 0,1088 \\
\hline $\mathrm{ALA}_{\mathrm{it}}$ & 0,6113 & 0,2448 & 0,4587 & 0,5894 & 0,7604 \\
\hline $\mathrm{GC}_{\text {it }}$ & 51,5266 & 19,1437 & 37,4472 & 50,9016 & 67,6993 \\
\hline
\end{tabular}

Legenda: ${ }^{*} \mathrm{p}<0,1 ;{ }^{* *} \mathrm{p}<0,05$; ${ }^{* *} \mathrm{p}<0,01$; RSC it: Responsabilidade Social Corporativa; MS_FBit: Mídia Social Facebook; MS_TWit: Mídia Social Twitter; TAMit: Tamanho; $\mathrm{CV}_{i t}$ : Crescimento das Vendas; ROA $A_{i t}$ : Rentabilidade

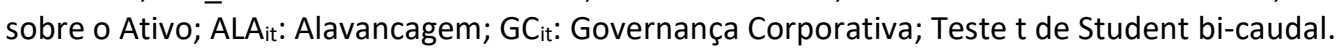

Fonte: Dados da pesquisa. 
Baseando-se na Tabela 2 e nas diferenças estatisticamente significantes evidenciadas pelo teste $t$ de médias, destina-se a análise inicial para as variáveis que se mostraram diferentes ao nível de $1 \%$. O Painel A demonstra que as empresas com engajamento no Facebook apresentam, em média, maiores níveis de $\mathrm{RSC}_{i t}$, bem como maior retorno sobre o ativo $\left(R O A_{i t}\right)$ e são mais alavancadas $\left(A L A_{i t}\right)$, entretanto, apresentam menor crescimento de vendas $\left(\mathrm{CV}_{\text {it }}\right)$.

No Painel B observa-se que as empresas que possuem engajamento no Twitter são empresas com maiores níveis de $\mathrm{RSC}_{i t}$, maiores em termos de tamanho (TAM ${ }_{\mathrm{it}}$ ) e possuem as melhores práticas de $\mathrm{GC}\left(\mathrm{GC}_{\mathrm{it}}\right)$. Além disso, a variável de $\mathrm{RSC}_{\text {it }}$ apresenta menor dispersão com relação à média, o que evidencia que o comportamento socialmente responsável das empresas que se engajam em MS se mostra mais homogêneo do que o grupo que não se engaja.

Com relação ao Painel $C$, percebe-se que empresas da amostra atendem em média mais da metade dos itens analisados pela base de dados, no que concerne ao disclosure voluntário de questões sociais e ambientais ( $\mathrm{RSC}_{i t}$ ) e aos mecanismos de $\mathrm{GC}\left(\mathrm{GC}_{i t}\right)$. Ademais, as empresas da amostra possuem, em média, um crescimento de $14 \%$ nas vendas de um ano para o outro, seus ativos retornam em forma de lucros cerca de $6 \%$ ao ano e para cada $R \$ 1,00$ de ativo total, as empresas possuem $\mathrm{R} \$ 0,60$ de capital de terceiros. Além disso, chama-se atenção para a dispersão do variável crescimento das vendas $\left(\mathrm{CV}_{\mathrm{it}}\right)$ e rentabilidade sobre o ativo, as quais evidenciam que existem empresas na amostra com valores relativamente pequenos (inclusive negativos) e relativamente elevados, o que é demonstrado pelos percentis 25 e 75 e pelo elevado desvio padrão.

A Tabela 3 apresenta a matriz de correlação (Pearson) entre as variáveis da pesquisa.

Tabela 3 - Correlação de Pearson

\begin{tabular}{|c|c|c|c|c|c|c|c|c|}
\hline Variável & MS_FBit & MS_TW & $\mathrm{RSC}_{\mathrm{it}}$ & TAM $_{\text {it }}$ & $\mathrm{CV}_{\text {it }}$ & ROA $_{i t}$ & ALA it & $\mathrm{GC}_{\mathrm{it}}$ \\
\hline MS_FB & 1 & & & & & & & \\
\hline MS_TW & $0,6070^{* * *}$ & 1 & & & & & & \\
\hline $\mathrm{RSC}_{\text {it }}$ & $0,4458 * * *$ & $0,4430 * * *$ & 1 & & & & & \\
\hline TAM $_{\text {it }}$ & $0,1894 * * *$ & $0,3893 * * *$ & $0,5282 * * *$ & 1 & & & & \\
\hline $\mathrm{CV}_{\text {it }}$ & $-0,1248 * * *$ & $-0,0887^{*}$ & $-0,1287^{* *}$ & $-0,1130 * *$ & 1 & & & \\
\hline $\mathrm{ROA}_{\text {it }}$ & $0,1805^{* * *}$ & $0,1252^{* *}$ & $0,2164 * * *$ & 0,0224 & $-0,0380$ & 1 & & \\
\hline ALA $_{i t}$ & $0,1401 * * *$ & 0,0443 & $0,1219 * *$ & 0,0562 & 0,0255 & $-0,3836^{*}$ & 1 & \\
\hline $\mathrm{GC}_{\text {it }}$ & $0,1968 * * *$ & $0,2349 * * *$ & $0,2200 * * *$ & 0,0306 & $-0,0320$ & $0,1473^{* * *}$ & $0,1450 * * *$ & 1 \\
\hline
\end{tabular}

Legenda: ${ }^{*} p<0,1 ;{ }^{* *} p<0,05 ;{ }^{* * *} p<0,01$; RSC ${ }_{i t:}$ Responsabilidade Social Corporativa; MS_FBit: Mídia Social Facebook; MS_TW sobre o Ativo; ALAit: Alavancagem; GC it: Governança Corporativa.

Fonte: Dados da pesquisa.

A matriz de correlação confirma o evidenciado pelo teste $t$ de médias entre empresas com e sem mídia social, ou seja, na matriz se pode verificar uma associação positiva e significativa ao nível de $1 \%$ entre o comportamento socialmente responsável ( $\mathrm{RSC}_{\text {it }}$ ) e o

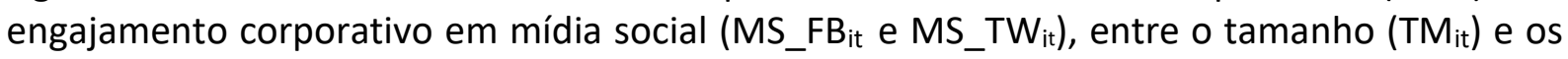
mecanismos de $\mathrm{GC}\left(\mathrm{GC}_{i t}\right)$ e as mídias sociais.

Com relação à associação entre RSC (variável dependente no modelo econométrico desta pesquisa) e as demais variáveis (variáveis explicativas), observa-se que todas apresentaram associações significantes ao nível de $1 \%$ e $5 \%$, o que reflete que o tamanho das empresas $\left(T A M_{i t}\right)$, o crescimento das vendas $\left(C V_{i t}\right)$, a rentabilidade sobre o ativo $\left(\right.$ ROA $\left._{i t}\right)$, a alavancagem ( $\left(A L A_{i t}\right)$ e a governança corporativa $\left(G C_{i t}\right)$ são possivelmente variáveis explicativas relevantes para o modelo econométrico. 
A Tabela 4 apresenta os resultados da influência do engajamento corporativo em mídias sociais (Facebook e Twitter) no comportamento socialmente responsável ( RSC $_{i t}$ ), foco desta pesquisa. Ressalta-se que os pressupostos da regressão linear múltipla (normalidade dos resíduos, auto correlação dos resíduos e multicolinearidade das variáveis) foram testados e não apresentaram problemas, conforme exposto na Tabela 4 pelos testes Jacques Bera (para normalidade dos resíduos), teste Durbin Watson (para auto correlação dos resíduos) e teste VIF (para multicolinearidade entre as variáveis).

Tabela 4 - Resultado da regressão RSC e mídia social

\begin{tabular}{|c|c|c|c|c|}
\hline \multirow{3}{*}{ Variáveis } & \multicolumn{4}{|c|}{ Variável Dependente: $\mathbf{R S C}_{\text {it }}$} \\
\hline & \multicolumn{2}{|c|}{ MS it: Facebook } & \multicolumn{2}{|c|}{ MS it: Twitter } \\
\hline & Coef. (Est. $t$ ) & Coef. (Est. $t$ ) & Coef. (Est. $t$ ) & Coef. (Est. $t$ ) \\
\hline Constante & $\begin{array}{c}47,46647^{* * *} \\
(12,27)\end{array}$ & $\begin{array}{c}-81,7441^{* * *} \\
(-6,45)\end{array}$ & $\begin{array}{c}50,68747^{* * *} \\
(12,08)\end{array}$ & $\begin{array}{c}-79,7781 * * * \\
(-5,68)\end{array}$ \\
\hline$M S_{i t}$ & $\begin{array}{c}143,8565^{* * *} \\
(10,51)\end{array}$ & $\begin{array}{c}98,71636 * * * \\
(7,66)\end{array}$ & $\begin{array}{c}20,91368 * * * \\
(8,06)\end{array}$ & $\begin{array}{c}10,8485^{* * * *} \\
(4,75)\end{array}$ \\
\hline TAM $_{\text {it }}$ & - & $\begin{array}{c}5,257033^{* * *} \\
(9,46)\end{array}$ & - & $\begin{array}{c}5,1487 * * * \\
(8,62)\end{array}$ \\
\hline $\mathrm{CV}_{\text {it }}$ & - & $\begin{array}{c}-1,016813 \\
(-1,25)\end{array}$ & - & $\begin{array}{c}-1,6415^{* *} \\
(-1,97)\end{array}$ \\
\hline ROA $_{\text {it }}$ & - & $\begin{array}{c}40,35088^{* * *} \\
(4,87)\end{array}$ & - & $\begin{array}{c}53,9049 * * * \\
(6,52)\end{array}$ \\
\hline $\mathrm{ALA}_{\text {it }}$ & - & $\begin{array}{c}10,36455^{* * *} \\
(2,71)\end{array}$ & - & $\begin{array}{c}16,7410 * * * \\
(4,33)\end{array}$ \\
\hline $\mathrm{GC}_{\text {it }}$ & - & $\begin{array}{c}0,0707401 \\
(1,64)\end{array}$ & - & $\begin{array}{c}0,0668 \\
(1,39)\end{array}$ \\
\hline Efeito Fixo de Setor e Ano & Sim & Sim & Sim & Sim \\
\hline Significância do modelo & $0,0000 * * *$ & $0,0000 * * *$ & $0,0000 * * *$ & $0,0000 * * *$ \\
\hline$R^{2}$ & 0,0537 & 0,0843 & 0,0377 & 0,0707 \\
\hline VIF & 1,07 & $1,08-1,68$ & 1,19 & $1,07-1,62$ \\
\hline Durbin Watson & 1,988861 & 1,98563 & 1,843428 & 1,9223 \\
\hline Jacques Bera & 0,0256 & 0,3365 & 0,0132 & 0,1952 \\
\hline Número de Observações & 360 & 360 & 360 & 360 \\
\hline \multicolumn{5}{|c|}{$\begin{array}{l}\text { Legenda: VIF: Variance Inflator Factor; Obs.: Observações; RSCit: Responsabilidade Social Corporativa; MSit: Mídia } \\
\text { Social; TAMit: Tamanho; } \mathrm{CV}_{\text {it: }} \text { Crescimento das Vendas; ROAit: Rentabilidade sobre o Ativo; ALAit: Alavancagem; } \\
\mathrm{GC}_{i t} \text { : Governança Corporativa; Coef.=Coeficiente; (Est. t)=Estatística } t \text { (entre parênteses); VIF: Variance Inflaction } \\
\text { Factor; }{ }^{*} \mathrm{p}<0,1 ;{ }^{* *} \mathrm{p}<0,05 ;{ }^{* *} \mathrm{p}<0,01 \text {. }\end{array}$} \\
\hline
\end{tabular}

Fonte: Elaborado pelos autores.

Na Tabela 4 é possível verificar que os modelos de regressão Tobit foram significativos e que as práticas de RSC são explicadas 5,37\% pelo Facebook e 3,77\% pelo Twitter, ainda quando inserida as variáveis de controle observa-se um aumento do poder explicativo de $3,06 \%$ e 3,30\%, respectivamente. O poder explicativo dos modelos Tobit operacionalizados nesta pesquisa foram ainda superiores ao evidenciado por Paulino, Silva e Girão (2018), os quais obtiveram modelos com pseudo $\mathrm{R}^{2}$ de $1,57 \%$ ao inserir o Twitter como variável explicativa como o disclosure voluntário e pseudo $R^{2}$ de $-4,27 \%$ ao inserir o Facebook.

Percebe-se, conforme o esperado, uma relação positiva e significativa entre o engajamento corporativo em ambas as mídias sociais e as práticas de RSC ( RSC $_{i t}$ ), o que revela que as empresas da amostra fazem uso corporativo do Facebook e Twitter como um meio de se engajar com suas partes interessadas. Dessa forma, entende-se que a demanda de 
informações em mídia social impulsiona as organizações a divulgarem informações sobre práticas socialmente responsáveis.

Esta análise atende ao sugerido por Manetti e Bellucci (2016), os quais por meio de uma análise de discurso de relatórios de sustentabilidade, destacaram a necessidade de estudos quantitativos sobre a relação entre a mídia social e relatórios de RSC. Além disso, este achado confirma empiricamente o destacado de forma conceitual por Lodhia e Stone (2017), os quais sugeriram que a internet possui ricos recursos e características capazes de possibilitar o aprimoramento na comunicação entre organizações e partes externas interessadas.

Com relação aos estudos empíricos, encontrou-se o de Paulino, Silva e Girão (2018) em âmbito brasileiro, o qual demonstrou que a quantidade de curtidas (no Facebook e no Twitter) possibilitou a ampliação do alcance informacional das organizações, uma vez que o número de curtidas reflete o feedback dos usuários, o qual se mostrou positivamente relacionado ao disclosure voluntário. Contudo, a relação entre o número de seguidores (no Facebook no Twitter) e o disclosure voluntário se mostrou negativa, o que contradiz o esperado pelos autores.

Dessa forma, esta pesquisa expande a literatura anterior de diversas maneiras. Primeiro, por acrescentar um trabalho empírico à literatura, a qual se mostra incipiente nessa temática, sendo que dentre os três estudos que abordaram a mídia social como um fator facilitador da divulgação voluntária de RSC, apenas o de Paulino, Silva e Girão (2018) é empírico. Além disso, esta pesquisa acrescenta uma evidência que reafirma os pressupostos teóricos de Paulino, Silva e Girão (2018) sobre a influência positiva do Facebook e Twitter na divulgação de práticas socialmente responsáveis, a qual se mostrou empiricamente negativa naquele estudo.

Em termos econômicos, ao comparar a média das práticas de RSC ( RSC $_{i t}$ ) de 55,7254 (Tabela 2) e o coeficiente de 143,8565 (Tabela 4) da variável $\mathrm{MS}_{\text {it }}$ - Facebook, nota-se um aumento das práticas de RSC divulgadas de cerca de $258 \%$ (143,8565: 55,7254) em empresas que possuem engajamento corporativo no Facebook. Em relação ao Twitter, observa-se que a RSC aumenta cerca de $38 \%(20,91368: 55,7254)$ com o uso desta mídia social.

Com base nos resultados das equações que contemplam as variáveis de controle, observa-se um aumento do $\mathrm{R}^{2}$ em ambos os modelos, quando comparados com os resultados dos modelos que apresentam somente a relação direta entre MS e RSC. As evidências indicam que as variáveis de controle adicionadas aos modelos contribuem para explicar a variável dependente, especificamente, o poder explicativo dos modelos aumentou 3,06\% e 3,30\%.

Em relação às variáveis de controle, o coeficiente do tamanho da empresa demonstrase positivo e significativo, indicando que as empresas maiores possuem mais práticas de RSC, o que é condizente com a literatura e também evidenciado por Ghoul et al. (2019). Referente ao retorno sobre os ativos, os achados confirmam o relatado por Ghoul et al. (2019), sendo evidenciado uma relação positiva e significativa, que indica que empresas mais lucrativas possuem maior RSC. Os resultados acerca da alavancagem corroboram o estudo de Chakroun, Matoussi e Mbirki (2017), os quais evidenciaram uma relação significativa e positiva, entretanto, divergem do estudo nacional de Paulino, Silva e Girão (2017), que não constataram relação significativa entre alavancagem e RSC.

Realizou-se o teste sensibilidade com a finalidade de oferecer robustez aos achados da análise principal. Dessa forma, foi utilizada como variável de interesse uma variável dummy do percentil $\geq 75$, referente aos scores de uso corporativo das mídias sociais. Assim, corresponde a 1 as empresas que tiveram score igual ou superior a 0,1304 no Facebook e 
EDIÇÃO ESPECIAL: COMEMORATIVA AOS 45 ANOS DOS CURSOS DE GRADUAÇÃO EM CIÊNCIAS CONTÁBEIS E ADMINISTRAÇÃO DA UNOCHAPECÓ

0,5738 no Twitter, sendo 0 caso contrário. A Tabela 5 apresenta os resultados do teste de sensibilidade.

Tabela 5 - Teste de sensibilidade

\begin{tabular}{|c|c|c|c|c|}
\hline \multirow{3}{*}{ Variáveis } & \multicolumn{4}{|c|}{ Variável Dependente: RSC $_{\text {it }}$} \\
\hline & \multicolumn{2}{|c|}{$\mathrm{MS}_{\text {it: }}$ Facebook } & \multicolumn{2}{|c|}{$\mathrm{MS}_{\text {it: }}$ Twitter } \\
\hline & Coef. (Est. $t$ ) & Coef. (Est. $t$ ) & Coef. (Est. $t$ ) & Coef. (Est. $t$ ) \\
\hline Constante & $\begin{array}{c}54,90286^{* * *} \\
(11,70)\end{array}$ & $\begin{array}{c}-99,51353^{* * *} \\
(-7,29)\end{array}$ & $\begin{array}{c}54,54305^{* * *} \\
(11,770\end{array}$ & $\begin{array}{c}-87,93699 * * * \\
(-5,85)\end{array}$ \\
\hline$M S_{i t}$ & $\begin{array}{c}10,17892^{* * *} \\
(5,16)\end{array}$ & $\begin{array}{c}3,925938^{* * *} \\
(2,19)\end{array}$ & $\begin{array}{c}12,00636 * * * \\
(7,16)\end{array}$ & $\begin{array}{c}4,516827^{* * *} \\
(2,85)\end{array}$ \\
\hline TAM $_{\text {it }}$ & - & $\begin{array}{c}6,097157^{* * *} \\
(10,60)\end{array}$ & - & $\begin{array}{c}5,557064^{* * *} \\
(8,82)\end{array}$ \\
\hline$C V_{\text {it }}$ & - & $\begin{array}{c}-1,707227^{*} \\
(-1,88)\end{array}$ & - & $\begin{array}{c}-1,849236^{* *} \\
(-2,06)\end{array}$ \\
\hline $\mathrm{ROA}_{\text {it }}$ & - & $\begin{array}{c}52,56115^{* * *} \\
(6,19)\end{array}$ & - & $\begin{array}{c}56,27796^{* * *} \\
(6,67)\end{array}$ \\
\hline $\mathrm{ALA}_{\text {it }}$ & - & $\begin{array}{c}14,83023^{* * * *} \\
(3,93)\end{array}$ & - & $\begin{array}{c}16,92773^{* * *} \\
(4,49)\end{array}$ \\
\hline $\mathrm{GC}_{\text {it }}$ & - & $\begin{array}{c}0,1019203^{* *} \\
(2,10)\end{array}$ & - & $\begin{array}{c}0,0896221^{*} \\
(1,80)\end{array}$ \\
\hline Efeito Fixo de Setor e Ano & Sim & $\operatorname{Sim}$ & Sim & Sim \\
\hline Significância do modelo & $0,0000 * * *$ & $0,0000 * * *$ & $0,0000 * * *$ & $0,0000 * * *$ \\
\hline $\mathrm{R}^{2}$ & 0,0256 & 0,0663 & 0,0297 & 0,0668 \\
\hline VIF & 1,26 & 1,07 & 1,09 & 1,07 \\
\hline Durbin Watson & 2,045373 & 2,018775 & 1,804831 & 1,936346 \\
\hline Jacques Bera & 0,0038 & 0,0789 & 0,0412 & 0,1226 \\
\hline Número de Observações & 360 & 360 & 360 & 360 \\
\hline \multicolumn{5}{|c|}{$\begin{array}{l}\text { Legenda: RSC } i t: \text { Responsabilidade Social Corporativa; } \mathrm{MS}_{\mathrm{it}} \text { : Mídia Social; TAMit: Tamanho; } \mathrm{CV}_{\mathrm{it}} \text { : Crescimento das } \\
\text { Vendas; ROAit: Rentabilidade sobre o Ativo; ALAit: Alavancagem; } \mathrm{GC}_{\mathrm{it}} \text { : Governança Corporativa Coef.=Coeficiente; } \\
\text { (Est. } t \text { )=Estatística } t \text { (entre parênteses); VIF: Variance Inflaction Factor; Sktest: Skewness and kurtosis test for } \\
\text { normality; Sig.: Significância; N.: Número; }{ }^{*} \mathrm{p}<0,1 ;{ }^{* *} \mathrm{p}<0,05 ;{ }^{* *} \mathrm{p}<0,01 .\end{array}$} \\
\hline
\end{tabular}

Fonte: Elaborado pelos autores.

Tratando-se dos modelos, ambos foram significativos, e com poder explicativo que varia entre $2,56 \%$ e $6,68 \%$, o que sugere as variáveis independentes e de controle são úteis para explicar as práticas de RSC. Observa-se na Tabela 5 que as mídias sociais (Facebook e Twitter) mensuradas pelo percentil 75 do score apresentaram relação positiva e significativa com as práticas de RSC. Pode-se depreender deste resultado que as empresas mais engajadas em mídias sociais possuem melhores práticas de RSC, possivelmente os usuários destes canais demandam ações organizacionais responsáveis, fato que pode impulsionar a RSC.

Os resultados evidenciados na Tabela 5 confirmam o apresentado na análise principal (Tabela 4), quanto à relação entre as mídias sociais e RSC. Dessa maneira, não se pode rejeitar a $\mathrm{H}_{1}$, que pressupõe que o uso corporativo de mídias sociais está positivamente relacionado com as práticas de RSC em empresas brasileiras. Desta forma, infere-se que as empresas fazem uso corporativo de mídias sociais, como Facebook e Twitter, como uma maneira de se engajar com suas partes interessadas.

De forma geral, infere-se que as mídias sociais do Facebook e Twitter podem ser consideradas um meio facilitador das empresas divulgarem informações voluntárias, o que de acordo com o exposto na literatura anterior (BLANKESPOOR; MILLER; WHITE, 2013), pode ser decorrente do fato de que a mídia social permite uma divulgação rápida, abrangente e de 
menor custo. Além disso, este estudo reafirma os pressupostos de Crisan e Zbuchea (2015), de que o Facebook e Twitter são plataformas eficazes na disseminação de informações e no engajamento entre partes interessadas e organizações.

\section{CONSIDERAÇÕES FINAIS}

Os principais achados desta pesquisa demonstram uma relação positiva entre o uso corporativo das mídias sociais e as práticas de RSC em empresas brasileiras, observadas durante os anos de 2013 a 2017. As variáveis de mídia social foram mensuradas por meio do engajamento das empresas no Facebook e Twitter, sendo operacionalizado um score de uso corporativo. Já a variável de RSC é composta por uma medida agregada que contempla práticas relacionadas ao uso de recursos, emissão de poluentes, poluição, força de trabalho, direitos humanos, comunidade e responsabilidade com os produtos.

$\mathrm{Na}$ análise principal, evidenciou-se uma relação positiva entre o uso corporativo de ambas as mídias sociais (Facebook e Twitter) e as práticas de RSC (a nível de 1\%). Adicionalmente, realizou-se um teste de sensibilidade, com o intuito de fornecer robustez aos resultados, sendo que a partir deste os achados obtidos na análise principal foram confirmados, o que permitiu a não rejeição da hipótese de pesquisa, pois, o uso corporativo de mídia social apresentou-se positivamente relacionado com a divulgação de práticas de responsabilidade social corporativa em empresas brasileiras.

De forma geral, os resultados sugerem que as organizações utilizam o Facebook e o Twitter como forma de se comunicarem com seus stakeholders, o que é compreendido pelo fato de as mídias sociais serem meios rápidos, menos custosos e abrangentes de comunicação e, por isso, possibilitam e facilitam o disclosure voluntário. Estas evidências geram contribuições adicionais para as pesquisas já realizadas, principalmente em contexto nacional, em relação ao engajamento corporativo em mídias sociais e às práticas de RSC, bem como, contribui com stakeholders que buscam informações acerca da RSC. Além disso, expande as pesquisas que relacionaram os temas por meio do teste empírico do modelo proposto, de forma a gerar evidencias adicionais à literatura.

Ainda, contribui-se ao analisar as informações referentes ao uso corporativo de mídias sociais de forma conjunta, por meio de um score, que visou mensurar o engajamento corporativo. Em relação à medida de RSC, contribui-se ao considerar o conceito teórico multidimensional da RSC, que compreende os pilares ambiental e social de práticas de RSC. Na prática, a pesquisa contribuiu ao evidenciar que o Facebook e Twitter, constituem-se como canais viáveis para o engajamento corporativo, ainda essas mídias sociais correspondem a uma fonte de informação adicional, tanto para empresas, quanto para investidores e demais interessados.

Pesquisas futuras podem analisar o conteúdo das publicações das empresas referente a RSC e a reação dos demais usuários de mídias sociais, por meio de comentários, curtidas e compartilhamentos. Adicionalmente, outras mídias sociais como o Instagram e Linkedln poderiam ser analisadas, bem como, as mídias sociais poderiam ser analisadas em conjunto, com o intuito de verificar se o a presença da empresa em mais de uma mídia social, infere em melhores práticas de RSC.

Como limitações, destaca-se inicialmente o fato de não analisar o conteúdo das publicações no Facebook e Twitter, e a questão de que as variáveis de mídia social, como número de tweets, seguidores, curtidas entre outras, foram coletadas em um período estático. Além disso, pode ser destacado como uma limitação a impossibilidade de generalizar 
EDIÇÃO ESPECIAL: COMEMORATIVA AOS 45 ANOS DOS CURSOS DE GRADUAÇÃO EM CIÊNCIAS CONTÁBEIS E ADMINISTRAÇÃO DA UNOCHAPECÓ

os resultados, visto que foram analisadas apenas as empresas listadas na $[B]^{3}$ com informações sobre práticas de RSC.

\section{REFERÊNCIAS}

AGOSTINO, D.; SIDOROVA, Y. How social media reshapes action on distant customers: some empirical evidence. Accounting, Auditing \& Accountability Journal, v. 30, n. 4, p. 777-794, 2017. DOI: https://doi.org/10.1108/AAAJ-07-2015-2136.

AININ, S.; PARVEEN, F.; MOGHAVVEMI, S.; JAAFAR, N. I.; MOHD SHUIB, N. L. Factors influencing the use of social media by SMEs and its performance outcomes. Industrial Management \& Data Systems, v. 115, n. 3, p. 570-588, 2015. DOI: https://doi.org/10.1108/IMDS-07-2014-0205.

ALI, I.; JIMENEZ-ZARCO, A. I.; BICHO, M. Using social media for CSR communication and engaging stakeholders. In: Corporate social responsibility in the digital age. Emerald Group Publishing Limited.v. 7 p. 165-185, 2015. DOI: https://doi.org/10.1108/S2043052320150000007010.

BLANKESPOOR, E.; MILLER, G. S.; WHITE, H. D. The role of dissemination in market liquidity: Evidence from firms' use of Twitter ${ }^{\mathrm{TM}}$. The Accounting Review, v. 89, n. 1, p. 79-112, 2013. DOI: https://doi.org/10.2308/accr-50576.

BRAUERS, W. K.; ZAVADSKAS, E. K. The MOORA method and its application to privatization in a transition economy. Control and Cybernetics, v. 35, p. 445-469, 2006.

CADE, N. L. Corporate social media: How two-way disclosure channels influence investors. Accounting, Organizations and Society, v. 68, p. 63-79, 2018. DOI: https://doi.org/10.1016/j.aos.2018.03.004.

CAO, Y.; AJJAN, H.; HONG, P.; LE, T. Using social media for competitive business outcomes: An empirical study of companies in China. Journal of Advances in Management Research, $v$. 15, n. 2, p. 211-235, 2018. DOI: https://doi.org/10.1108/JAMR-05-2017-0060.

CHAHINE, S; MALHOTRA, N. K. Impact of social media strategies on stock price: the case of Twitter. European Journal of Marketing, v. 52, n. 7/8, p. 1526-1549, 2018. DOI: https://doi.org/10.1108/EJM-10-2017-0718.

CHANDA, R.; ZAORSKI, S. Social media usage in the financial services industry: Toward a business-driven compliance approach. Journal of Taxation \& Regulation of Financial Institutions, v. 26, n. 5, p. 5-20, 2013.

CHAKROUN, R.; MATOUSSI, H.; MBIRKI, S. Determinants of CSR disclosure of Tunisian listed banks: a multi-support analysis. Social Responsibility Journal, v. 13, n. 3, p. 552-584, 2017. DOI: https://doi.org/10.1108/SRJ-04-2016-0055. 
CRIȘAN, C; ZBUCHEA, A. CSR and Social Media: Could Online Repositories Become Regulatory Tools for CSR Related Activities' Reporting? In: Corporate social responsibility in the digital age. Emerald Group Publishing Limited, 2015. p. 197-219. DOI: https://doi.org/10.1108/S2043-052320150000007011.

DLAMINI, N. N.; JOHNSTON, K. The use of social media by South African organisations. Journal of Advances in Management Research, v. 15, n. 2, p. 198-210, 2018. DOI: https://doi.org/10.1108/JAMR-05-2017-0063.

DRAKE, M. S.; ROULSTONE, D. T.; THORNOCK, J. R. Investor information demand: Evidence from Google searches around earnings announcements. Journal of Accounting research, $v$. 50, n. 4, p. 1001-1040, 2012. DOI: https://doi.org/10.1111/j.1475-679X.2012.00443.x.

DYE, R. A. An evaluation of "essays on disclosure" and the disclosure literature in accounting. Journal of accounting and economics, v. 32, n. 1-3, p. 181-235, 2001. DOI: https://doi.org/10.1016/S0165-4101(01)00024-6.

FERRER, E.; BOUSOÑO, C.; JORGE, J.; LORA, L.; MIRANDA, E.; NATALIZIO, N. Enriching social capital and improving organizational performance in the age of social networking. Business and Management, v. 5, n. 2, p. 94-281, 2013.

GHOUL, S. E.; GUEDHAMI, O.; NASH, R.; PATEL, A. New evidence on the role of the media in corporate social responsibility. Journal of Business Ethics, v. 154, n. 4, p. 1051-1079, 2019. DOI: https://doi.org/10.1007/s10551-016-3354-9.

HE, W.; WANG, F.; AKULA, V. Managing extracted knowledge from big social media data for business decision making. Journal of Knowledge Management, v. 21, n. 2, p. 275-294, 2017. DOI: https://doi.org/10.1108/JKM-07-2015-0296.

JUNG, M. J.; NAUGHTON, J. P.; TAHOUN, A.; WANG, C. Do firms strategically disseminate? Evidence from corporate use of social media. The Accounting Review, v. 93, n. 4, p. 225-252, 2018. DOI: https://doi.org/10.2308/accr-51906.

KAPLAN, A. M.; HAENLEIN, M. Users of the world, unite! The challenges and opportunities of Social Media. Business horizons, v. 53, n. 1, p. 59-68, 2010. DOI:

https://doi.org/10.1016/j.bushor.2009.09.003.

LODHIA, S.; STONE, G. Integrated reporting in an internet and social media communication environment: conceptual insights. Australian Accounting Review, v. 27, n. 1, p. 17-33, 2017. DOI: https://doi.org/10.1111/auar.12143.

MANETTI, G.; BELLUCCI, M. The use of social media for engaging stakeholders in sustainability reporting. Accounting, Auditing \& Accountability Journal, v. 29, n. 6, p. $985-$ 1011, 2016. DOI: https://doi.org/10.1108/AAAJ-08-2014-1797.

MANETTI, G.; BELLUCCI, M.; BAGNOLI, L. Stakeholder engagement and public information through social media: a study of Canadian and American public transportation agencies. The 
American Review of Public Administration, v. 47, n. 8, p. 991-1009, 2017. DOI: https://doi.org/10.1177/0275074016649260.

MILLER, G. S.; SKINNER, D. J. The evolving disclosure landscape: How changes in technology, the media, and capital markets are affecting disclosure. Journal of Accounting Research, $\mathrm{v}$. 53, n. 2, p. 221-239, 2015. DOI: https://doi.org/10.1111/1475-679X.12075.

PANIAGUA, J.; SAPENA, J. Business performance and social media: Love or hate? Business horizons, v. 57, n. 6, p. 719-728, 2014. DOI: https://doi.org/10.1016/j.bushor.2014.07.005.

PAULINO, G. C.; SILVA, G. S.; GIRÃO, L. F. A. P. Disclosure Voluntário Via Redes Sociais das Empresas Listadas no IBRX100. Revista Universo Contábil, v. 14, n. 1, p. 149-167, 2019. DOI: http://dx.doi.org/10.4270/ruc.2018324.

PRAHALAD, C. K.; RAMASWAMY, V. The future of competition: Co-creating unique value with customers. Harvard Business Press, 2004.

RODRIGUEZ, M.; PETERSON, R. M.; AJJAN, H. CRM/social media technology: impact on customer orientation process and organizational sales performance. In: Ideas in Marketing: Finding the New and Polishing the Old. Springer, Cham, 2015. p. 636-638. DOI:

https://doi.org/10.1007/978-3-319-10951-0_233.

ZHANG, J. Voluntary information disclosure on social media. Decision Support Systems, v. 73, p. 28-36, 2015. DOI: https://doi.org/10.1016/j.dss.2015.02.018.

ZHAO, H.; ZHANG, F.; KWON, J. Corporate social responsibility research in international business journals: An author co-citation analysis. International Business Review, v. 27, n. 2, p. 389-400, 2018. DOI: https://doi.org/10.1016/j.ibusrev.2017.09.006. 